\title{
A Bayesian Joint Dispersion Model With Flexible Links
}

\author{
Rui Martins
}

CENTRO

DE INVESTIGAÇÃO

INTERDISCIPUNAR

EGAS MONIZ

Egas Moniz Health School

Egas Moniz Interdisciplinary Research Center, Portugal 


\section{Summary}

(1) Background

- Longitudinal studies

- Outline

(2) HIV/AIDS Application

- Data

- Exploratory analysis

- Joint Model

(3) Results 


\section{Questions of interest}

- Biomarker, $Y_{1}$

- e.g. CD4 counts, collected repeatedly over time (longitudinal data)

- time to an event of interest, $Y_{2}$

- e.g. death from any cause (survival data)

- Separate Analysis

- Joint Analysis

what is the effect of the missing information due to drop-out in
assessing the trends of the repeated measures?
what is the effect of the longitudinal evolution of CD4 cell count in
the hazard rate for death? 


\section{Questions of interest}

- Biomarker, $Y_{1}$

- e.g. CD4 counts, collected repeatedly over time (longitudinal data)

- time to an event of interest, $Y_{2}$

- e.g. death from any cause (survival data)

- Separate Analysis

- does treatment affect survival?

- are the average longitudinal evolutions different between males and females?

- Joint Analysis 


\section{Questions of interest}

- Biomarker, $Y_{1}$

- e.g. CD4 counts, collected repeatedly over time (longitudinal data)

- time to an event of interest, $Y_{2}$

- e.g. death from any cause (survival data)

- Joint Analysis

- what is the effect of the missing information due to drop-out in assessing the trends of the repeated measures?

- what is the effect of the longitudinal evolution of CD4 cell count in the hazard rate for death? 


\section{Correlated data in Iongitudinal studies}
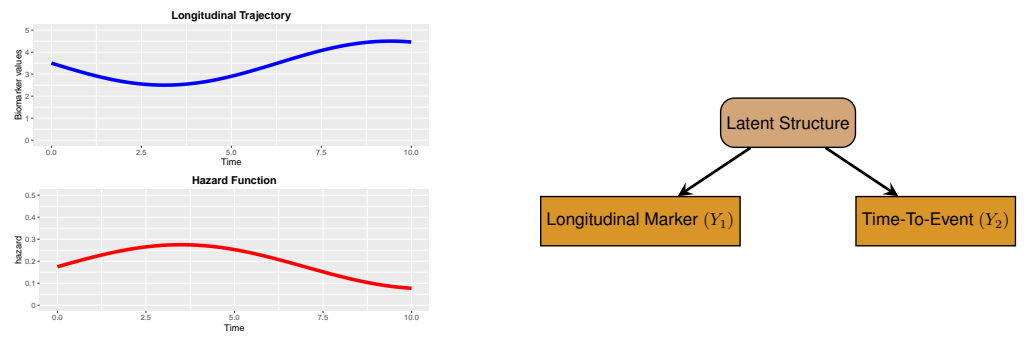

If the two processes are associated $\Rightarrow$ define a model for their joint probability distribution: $f\left(y_{1}, y_{2}\right)$ 


\section{Analysing HIV/AIDS data through} A Bayesian Joint Dispersion Model with Flexible Links 
- network of 88 laboratories located in every state in Brazil during Jan 2002 - Dec 2006;

- Sample: $n=500$ individuals; 2757 repeated measurements;

- Outcomes: $\mathrm{CD}^{+}{ }^{+} \mathrm{T}$ lymphocyte counts, $Y$, and time-to-death, $T$;

- Covariates: age $(<50=0, \geq 50=1)$; sex (Female $=0$, Male $=1)$; PrevOI (previous opportunistic infection at study entry=1, no previous infection=0); measurement times; date of diagnosis; date of death; failure indicator, $\delta$;

- Patients: 34 deaths. $88 \%$ between 15 and 49 years old; $60 \%$ males. $61 \%$ no previous infection. Initial CD4 median: 269 cells $/ \mathrm{mm}^{3}$ (men - 250 cells $/ \mathrm{mm}^{3}$; women - 295 cells $/ \mathrm{mm}^{3}$ ). 


\section{Longitudinal outcome}

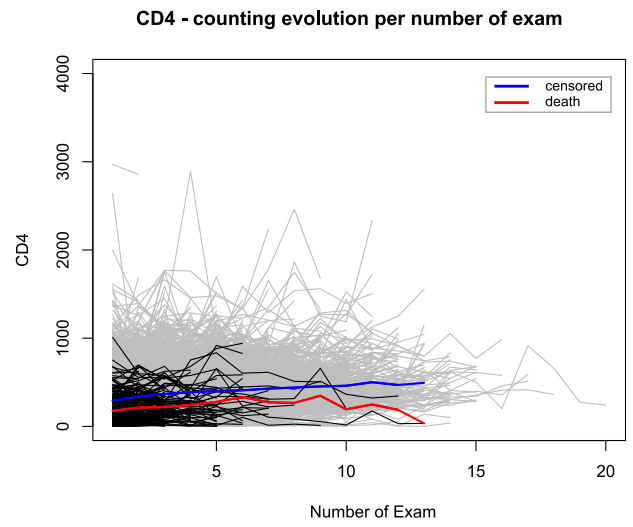




\section{Intra-individual variance (dispersion)}

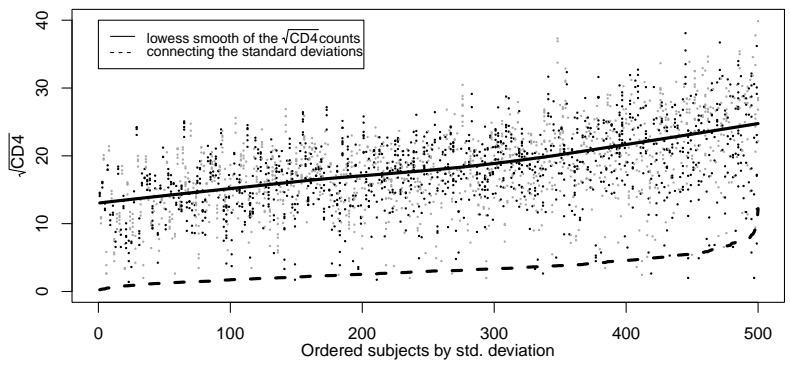

Individuals values of the $\sqrt{\mathrm{CD} 4}$ vs Std. Deviation (ordered) suggests considerable within-subject variance heterogeneity. Individuals with higher $\sqrt{\mathrm{CD} 4}$ values are associated with a higher variability. 


\section{Longitudinal specification}

- Mixed-effects dispersion model (McLain et al. 2012)

$$
\begin{aligned}
y_{i j} \mid \boldsymbol{b}_{i}, \sigma_{i}^{2} & \sim \mathcal{N}\left(m_{i}\left(t_{i j}\right), \sigma_{i}^{2}\right), \quad j=1, \ldots, n_{i} \\
m_{i}\left(t_{i j}\right) & =\boldsymbol{\beta}_{1}^{\top} \mathbf{x}_{1 i}\left(t_{i j}\right)+\boldsymbol{b}_{1 i}^{\top} \mathbf{w}_{1 i}\left(t_{i j}\right), \\
\sigma_{i}^{2} & =\sigma_{0}^{2} \exp \left\{\boldsymbol{\beta}_{2}^{\top} \mathbf{x}_{2 i}\left(t_{i j}\right)+\boldsymbol{b}_{2 i}^{\top} \mathbf{w}_{2 i}\left(t_{i j}\right)\right\},
\end{aligned}
$$




\section{Longitudinal specification}

- Mixed-effects dispersion model (McLain et al. 2012)

$$
\begin{aligned}
y_{i j} \mid \boldsymbol{b}_{i}, \sigma_{i}^{2} & \sim \mathcal{N}\left(m_{i}\left(t_{i j}\right), \sigma_{i}^{2}\right), \quad j=1, \ldots, n_{i} \\
m_{i}\left(t_{i j}\right) & =\boldsymbol{\beta}_{1}^{\top} \mathbf{x}_{1 i}\left(t_{i j}\right)+\boldsymbol{b}_{1 i}^{\top} \mathbf{w}_{1 i}\left(t_{i j}\right), \\
\sigma_{i}^{2} & =\sigma_{0}^{2} \exp \left\{\boldsymbol{\beta}_{2}^{\top} \mathbf{x}_{2 i}\left(t_{i j}\right)+\boldsymbol{b}_{2 i}^{\top} \mathbf{w}_{2 i}\left(t_{i j}\right)\right\},
\end{aligned}
$$

- $\mathbf{y}_{i}=\left(y_{i 1}, \ldots, y_{i n_{i}}\right) \rightarrow n_{i}$ observed repeated measures, $\sqrt{\text { CD4 }}$

- $\mathbf{t}_{i}=\left(t_{i 1}, \ldots, t_{i n_{i}}\right) \rightarrow$ visiting times

- $\mathbf{x}_{1 i}, \mathbf{x}_{2 i}, \mathbf{w}_{1 i}$ and $\mathbf{w}_{2 i} \rightarrow$ individual covariates (time-dependent?)

- $\boldsymbol{\beta}_{1}$ and $\boldsymbol{\beta}_{2} \rightarrow$ population parameters

- $\left(\boldsymbol{b}_{1 i}^{\top}, \boldsymbol{b}_{2 i}^{\top}\right)=\boldsymbol{b}_{i} \mid \Sigma \sim \mathcal{N}_{p}(\mathbf{0}, \Sigma) \rightarrow$ time-independent random-effects 


\section{Longitudinal outcome}

$$
\sqrt{\mathrm{CD}}_{i j} \mid \boldsymbol{b}_{i}, \sigma_{i}^{2} \sim \mathcal{N}\left(m_{i}\left(t_{i j}\right), \sigma_{i}^{2}\right)
$$

- Longitudinal mean

$$
m_{i}\left(t_{i j}\right)=\beta_{10}+\beta_{11} \text { sex }_{i}+\beta_{12} \text { age }_{i}+\beta_{13} \text { PrevOl }_{i}+\beta_{14} t_{i j}+b_{1 i, 1}+b_{1 i, 2} t_{i j}
$$

- Dispersion model (3) may assume:

$$
\begin{aligned}
\sigma_{i}^{2} & =\sigma_{0}^{2} \exp \left\{\beta_{21} \text { sex }+\beta_{22} \text { age }+\beta_{23} \text { PrevOl }+b_{2 i}\right\} \\
\sigma_{i}^{2} & =\sigma_{0}^{2} \exp \left\{b_{2 i}\right\} \\
\sigma_{i}^{2} & \\
\sigma_{i}^{2} & =\sigma_{0}^{2} .
\end{aligned}
$$




\section{Longitudinal outcome}

$$
\sqrt{\mathrm{CD}}_{i j} \mid \boldsymbol{b}_{i}, \sigma_{i}^{2} \sim \mathcal{N}\left(m_{i}\left(t_{i j}\right), \sigma_{i}^{2}\right)
$$

- Longitudinal mean

$$
m_{i}\left(t_{i j}\right)=\beta_{10}+\beta_{11} \text { sex }_{i}+\beta_{12} \text { age }_{i}+\beta_{13} \text { PrevOl }_{i}+\beta_{14} t_{i j}+b_{1 i, 1}+b_{1 i, 2} t_{i j}
$$

- Dispersion model (3) may assume:

$$
\begin{aligned}
& \sigma_{i}^{2}=\sigma_{0}^{2} \exp \left\{\beta_{21} \text { sex }+\beta_{22} \text { age }+\beta_{23} \text { PrevOl }+b_{2 i}\right\}, \\
& \sigma_{i}^{2}=\sigma_{0}^{2} \exp \left\{b_{2 i}\right\}, \\
& \sigma_{i}^{2} \\
& \sigma_{i}^{2}=\sigma_{0}^{2} .
\end{aligned}
$$

\section{- Priors}

- $\beta_{1 p} \sim \mathcal{N}(0,100) ; p=0, \ldots, 4$ and $\beta_{2 q} \sim \mathcal{N}(0,100) ; q=1, \ldots, 3$

- $\boldsymbol{b}_{i} \mid \Sigma \sim \mathcal{N}_{p}(\mathbf{0}, \Sigma) ; \quad \Sigma^{-1} \sim \mathcal{W} i s h(R, \xi)$

- $\log \left(\sigma_{0}\right) \sim \mathcal{U}(-100,100)$; or $\log \left(\sigma_{i}\right) \sim \mathcal{U}(-100,100)$

- Other options: $1 / \sigma_{0}^{2} \sim \mathcal{G}(\epsilon, \epsilon)$ and $\sigma_{0} \mid \varpi \sim \mathrm{h}-\mathcal{C}(\varpi), \varpi \sim \mathcal{U}(0,100)$. 


\section{Survival specification}

- Time-dependent coefficients (Penalized cubic B-Splines)

$$
h_{i}\left(t \mid \boldsymbol{b}_{i}, \sigma_{i}\right)=h_{0}(t) \exp \left\{\boldsymbol{\beta}_{3}^{\top} \mathbf{x}_{3 i}+\mathcal{C}_{i}\left\{\boldsymbol{b}_{i}, \sigma_{i} ; \boldsymbol{g}(t)\right\}\right\}=h_{0}(t) \exp \left\{\varrho_{i}(t)\right\}
$$




\section{Survival specification}

- Time-dependent coefficients (Penalized cubic B-Splines)

$$
h_{i}\left(t \mid \boldsymbol{b}_{i}, \sigma_{i}\right)=h_{0}(t) \exp \left\{\boldsymbol{\beta}_{3}^{\top} \mathbf{x}_{3 i}+\mathcal{C}_{i}\left\{\boldsymbol{b}_{i}, \sigma_{i} ; \boldsymbol{g}(t)\right\}\right\}=h_{0}(t) \exp \left\{\varrho_{i}(t)\right\}
$$

- $\mathcal{C}_{i}\{.\} \rightarrow$ specifies which components of the longitudinal process are related to $h_{i}($.

- Link $\rightarrow$ Shared parameters

- $\boldsymbol{b}_{i}, \sigma_{i}$ 


\section{Survival specification}

- Time-dependent coefficients (Penalized cubic B-Splines)

$$
h_{i}\left(t \mid \boldsymbol{b}_{i}, \sigma_{i}\right)=h_{0}(t) \exp \left\{\boldsymbol{\beta}_{3}^{\top} \mathbf{x}_{3 i}+\mathcal{C}_{i}\left\{\boldsymbol{b}_{i}, \sigma_{i} ; \boldsymbol{g}(t)\right\}\right\}=h_{0}(t) \exp \left\{\varrho_{i}(t)\right\}
$$

- $\mathbf{x}_{3 i} \rightarrow$ baseline covariates

- $\beta_{3} \rightarrow$ population parameters

- $h_{0}(t) \rightarrow$ parametric (e.g. Weibull); P-Splines; Piecewise constant function.

- $\boldsymbol{g}(t)=\left(g_{1}(t), \ldots, g_{L}(t)\right) \rightarrow$ suitable vector of smooth functions (P-Splines) representing the time-dependent coefficients 


\section{Time-to-death}

$$
h_{i}\left(t \mid \boldsymbol{b}_{i}, \sigma_{i}\right)=h_{0}(t) \exp \left\{\boldsymbol{\beta}_{3}^{\top} \mathbf{x}_{3 i}+\mathcal{C}_{i}\left\{\boldsymbol{b}_{i}, \sigma_{i} ; \boldsymbol{g}(t)\right\}\right\}=h_{0}(t) \exp \left\{\varrho_{i}(t)\right\}
$$

- all models

$$
\boldsymbol{\beta}_{3}^{\top} \mathbf{x}_{3 i}=\beta_{31} \text { sex }_{i}+\beta_{32} \text { age }_{i}+\beta_{33} \text { PrevOl }_{i}
$$

o $C_{i}($.$) may assume:$

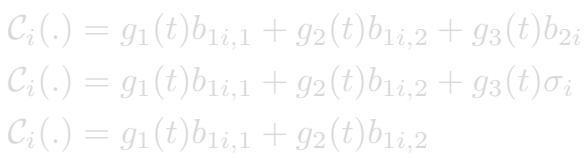




\section{Time-to-death}

$$
h_{i}\left(t \mid \boldsymbol{b}_{i}, \sigma_{i}\right)=h_{0}(t) \exp \left\{\boldsymbol{\beta}_{3}^{\top} \mathbf{x}_{3 i}+\mathcal{C}_{i}\left\{\boldsymbol{b}_{i}, \sigma_{i} ; \boldsymbol{g}(t)\right\}\right\}=h_{0}(t) \exp \left\{\varrho_{i}(t)\right\}
$$

all models

- $\mathcal{C}_{i}($.$) may assume:$

$$
\begin{aligned}
& \mathcal{C}_{i}(.)=g_{1}(t) b_{1 i, 1}+g_{2}(t) b_{1 i, 2}+g_{3}(t) b_{2 i} \\
& \mathcal{C}_{i}(.)=g_{1}(t) b_{1 i, 1}+g_{2}(t) b_{1 i, 2}+g_{3}(t) \sigma_{i} \\
& \mathcal{C}_{i}(.)=g_{1}(t) b_{1 i, 1}+g_{2}(t) b_{1 i, 2}
\end{aligned}
$$

\section{- 3 scenarios for $h_{0}(t)=\log \left(g_{0}(t)\right) \rightarrow$ Weibull, Penalized Splines}




\section{Time-to-death}

$$
h_{i}\left(t \mid \boldsymbol{b}_{i}, \sigma_{i}\right)=h_{0}(t) \exp \left\{\boldsymbol{\beta}_{3}^{\top} \mathbf{x}_{3 i}+\mathcal{C}_{i}\left\{\boldsymbol{b}_{i}, \sigma_{i} ; \boldsymbol{g}(t)\right\}\right\}=h_{0}(t) \exp \left\{\varrho_{i}(t)\right\}
$$

all models

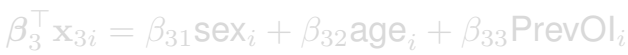

- $C_{i}($.$) may assume:$

- $g_{1}(t), g_{2}(t), g_{3}(t) \rightarrow$ Penalized Splines with 19 internal knots.

- $g_{l}(t)=\sum_{q=1}^{19} \gamma_{l q} B_{l q}(t), \quad l=1,2,3$

- $\gamma_{l 1} \sim \mathcal{N}(0,1000), \quad \gamma_{l q} \mid \tau_{l}^{2} \sim \mathcal{N}\left(\gamma_{l, q-1}, \tau_{l}^{2}\right), \quad q=2, \ldots, 19$

- $1 / \tau_{l}^{2} \sim \mathcal{G}(0.001,0.001), l=0,1,2,3$. 


\section{Time-to-death}

$$
h_{i}\left(t \mid \boldsymbol{b}_{i}, \sigma_{i}\right)=h_{0}(t) \exp \left\{\boldsymbol{\beta}_{3}^{\top} \mathbf{x}_{3 i}+\mathcal{C}_{i}\left\{\boldsymbol{b}_{i}, \sigma_{i} ; \boldsymbol{g}(t)\right\}\right\}=h_{0}(t) \exp \left\{\varrho_{i}(t)\right\}
$$

\section{all models}

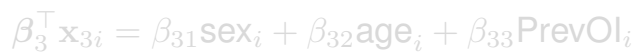

- $C_{i}($.$) may assume:$

- 3 scenarios for $h_{0}(t)=\log \left(g_{0}(t)\right) \rightarrow$ Weibull, Penalized Splines or piecewise constant 


\section{Joint likelihood}

We consider:

- $\mathbf{y}_{i}\left|\boldsymbol{b}_{i} \perp T_{i}\right| \boldsymbol{b}_{i} ; \quad y_{i j}\left|\boldsymbol{b}_{i} \perp y_{i l}\right| \boldsymbol{b}_{i}, j \neq l$

- non-informative right censoring

$$
L(\boldsymbol{\theta}, \mathbf{b}, \boldsymbol{\sigma} \mid \mathcal{D})=\prod_{i=1}^{N}\left(\prod_{j=1}^{n_{i}} p\left(y_{i}\left(t_{i j}\right) \mid \boldsymbol{\theta}, \boldsymbol{b}_{i}, \sigma_{i}^{2}\right)\right) p\left(T_{i}, \delta_{i} \mid \boldsymbol{\theta}, \boldsymbol{b}_{i}, \sigma_{i}\right)
$$

where

- $\mathcal{D}=\left\{\mathcal{D}_{i}\right\}_{i=1}^{N}=\left\{\left(\mathbf{y}_{i}, \mathbf{t}_{i}, T_{i}, \delta_{i}\right)\right\}_{i=1}^{N} \rightarrow$ observed data for the $N$ independent individuals

- $\boldsymbol{\theta} \rightarrow$ other parameters;

- $p(.) \rightarrow$ suitable density function 


\section{Joint likelihood}

We consider:

- $\mathbf{y}_{i}\left|\boldsymbol{b}_{i} \perp T_{i}\right| \boldsymbol{b}_{i} ; \quad y_{i j}\left|\boldsymbol{b}_{i} \perp y_{i l}\right| \boldsymbol{b}_{i}, j \neq l$

- non-informative right censoring

$$
L(\boldsymbol{\theta}, \mathbf{b}, \boldsymbol{\sigma} \mid \mathcal{D})=\prod_{i=1}^{N}\left(\prod_{j=1}^{n_{i}} p\left(y_{i}\left(t_{i j}\right) \mid \boldsymbol{\theta}, \boldsymbol{b}_{i}, \sigma_{i}^{2}\right)\right) p\left(T_{i}, \delta_{i} \mid \boldsymbol{\theta}, \boldsymbol{b}_{i}, \sigma_{i}\right)
$$

where

$$
p\left(y_{i}\left(t_{i j}\right) \mid \boldsymbol{\theta}, \boldsymbol{b}_{i}, \sigma_{i}^{2}\right)=\frac{1}{\sqrt{2 \pi \sigma_{i}^{2}}} \exp \left\{-\frac{\left[y_{i}\left(t_{i j}\right)-m_{i}\left(t_{i j}\right)\right]^{2}}{2 \sigma_{i}^{2}}\right\}
$$




\section{Joint likelihood}

We consider:

- $\mathbf{y}_{i}\left|\boldsymbol{b}_{i} \perp T_{i}\right| \boldsymbol{b}_{i} ; \quad y_{i j}\left|\boldsymbol{b}_{i} \perp y_{i l}\right| \boldsymbol{b}_{i}, j \neq l$

- non-informative right censoring

$$
L(\boldsymbol{\theta}, \mathbf{b}, \boldsymbol{\sigma} \mid \mathcal{D})=\prod_{i=1}^{N}\left(\prod_{j=1}^{n_{i}} p\left(y_{i}\left(t_{i j}\right) \mid \boldsymbol{\theta}, \boldsymbol{b}_{i}, \sigma_{i}^{2}\right)\right) p\left(T_{i}, \delta_{i} \mid \boldsymbol{\theta}, \boldsymbol{b}_{i}, \sigma_{i}\right)
$$

where

$$
\begin{aligned}
p\left(T_{i}, \delta_{i} \mid \boldsymbol{\theta}, \boldsymbol{b}_{i}, \sigma_{i}\right)= & h\left(T_{i} \mid \boldsymbol{\theta}, \boldsymbol{b}_{i}, \sigma_{i}\right)^{\delta_{i}} \times S\left(T_{i} \mid \boldsymbol{\theta}, \boldsymbol{b}_{i}, \sigma_{i}\right) \\
= & {\left[h_{0}\left(T_{i}\right) \exp \left\{\boldsymbol{\beta}_{3}^{\top} \mathbf{x}_{3 i}+\mathcal{C}_{i}\left\{\boldsymbol{b}_{i}, \sigma_{i} ; \boldsymbol{g}(t)\right\}\right\}\right]^{\delta_{i}} \times } \\
& \exp \left\{-\int_{0}^{T_{i}} h_{0}(u) \exp \left\{\boldsymbol{\beta}_{3}^{\top} \mathbf{x}_{3 i}+\mathcal{C}_{i}\left\{\boldsymbol{b}_{i}, \sigma_{i} ; \boldsymbol{g}(t)\right\}\right\} d u\right\}
\end{aligned}
$$




\section{Models comparison}

MCMC simulation within WinBUGS.

\begin{tabular}{|c|c|c|c|c|c|}
\hline \multicolumn{2}{|c|}{ Longitudinal model } & \multicolumn{4}{|c|}{ Survival model } \\
\hline \multirow[t]{2}{*}{$\overline{m_{i}}$} & \multirow[t]{2}{*}{$\overline{\sigma_{i}^{2}}$} & \multirow{2}{*}{$\varrho_{i}(t)$} & \multicolumn{3}{|c|}{$h_{0}$} \\
\hline & & & Weibull & P-Spline & Piecewise \\
\hline (4) & (5) & & 14671 & 12573 & 14317 \\
\hline (4) & (6) & $(11)+(12)$ & 14700 & 12848 & 14483 \\
\hline (4) & (5) & & 14307 & 12605 & 13365 \\
\hline (4) & (6) & & 14452 & 12917 & 13571 \\
\hline (4) & (7) & $(11)+(13)$ & 13134 & 12104 s & 12921 \\
\hline (4) & (8) & & 13956 & 12887 & 13533 \\
\hline (4) & (5) & & 14811 & 13334 & 14463 \\
\hline (4) & (6) & & 14923 & 13688 & 14599 \\
\hline (4) & (7) & + & 14314 & 13144 & 13968 \\
\hline (4) & (8) & & 14627 & 13553 & 14355 \\
\hline (4) & (8) & $(11)+g_{1} b_{1 i, 1}+g_{2} b_{1 i, 2}$ & 16984 & 15779 & 16383 \\
\hline
\end{tabular}

Tabela: WAIC values for the 33 joint models.

Best fit $\Rightarrow$ share the individual random-effects and the individual std-deviation considered as a covariate for the hazard model (Model $\mathbf{p})$. The heteroscedasticity is related to the survival time. 


\section{Posterior estimates for the time-dependent coefficients}
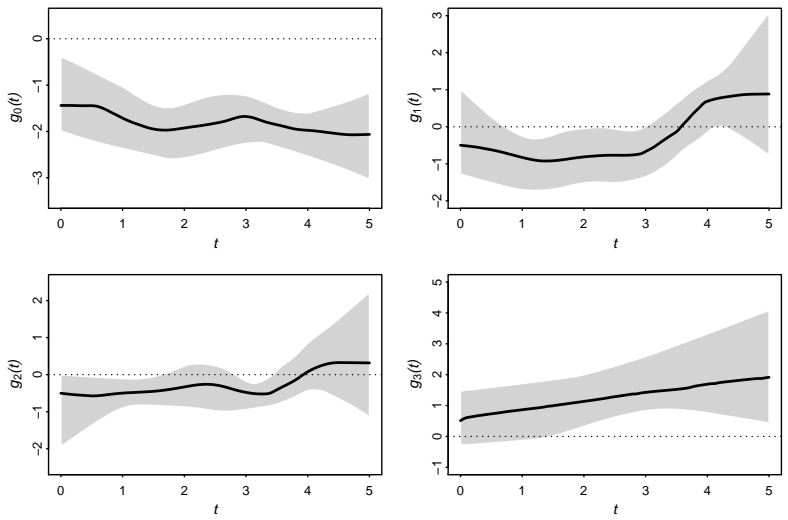

Figure 1: Posterior mean estimates, together with the corresponding 95\% Credible Bands (CB), for the selected model $\boldsymbol{\alpha}$. The top left panel shows $g_{0}=\log \left(h_{0}\right)$ and the subsequent panels have the time-varying regression coefficients as a function of time in years, $t$. 
BREZGER, A., AND LANG, S.

Simultaneous probability statements for bayesian p-splines.

Statistical Modelling 8, 2 (2008), 141-168

FAUCETT, C. L., AND THOMAS, D. C

Simultaneously modelling censored survival data and repeatedly measured covariates: a gibbs sampling approach.

Statistics in Medicine 15, 15 (Aug 1996), 1663-1685

IBRAHIM, J. G., ChEN, M. H., AND SINHA, D.

Bayesian Survival Analysis.

Springer-Verlag, 2001

LANG, S., AND BREZGER, A.

Bayesian p-splines.

Journal of Computational and Graphical Statistics 13, 1 (2004), 183-212

LUnN, D., Thomas, A., Best, N., ANd SPIEgelhalter, D.

Winbugs - a bayesian modelling framework: Concepts, structure, and extensibility.

Statistics and Computing 10 (2000), 325-337.

McLain, A. C., LUM, K. J., AND Sundaram, R.

A joint mixed effects dispersion model for menstrual cycle length and time-to-pregnancy.

Biometrics 68, 2 (Feb 2012), 648-656

Rizopoulos, D.

Joint Models for Longitudinal and Time-to-Event Data With Applications in R.

Chapman and Hall/CRC, 2012

\section{Thank you!}

